\title{
Electron Enhanced Raman Scattering and Its Applications in Solution Chemistry
}

\author{
Hiroharu YUI*,** \\ *Department of Chemistry, Faculty of Science, Tokyo University of Science, \\ 12 Funagawaramachi, Ichigaya, Shinjyuku, Tokyo 162-0826, Japan \\ **PRESTO, Japan Science and Technology Agency, 4-1-8 Honcho, Kawaguchi, Saitama 332-0012, Japan
}

\begin{abstract}
The present review describes a new enhancement technique for Raman scattering in aqueous solutions. Raman scattering spectroscopy has an inherent ability to distinguish between molecules with great similarity and provides useful information on local physical and chemical environments at their functional groups' level. Since the Raman scattering signals from water molecules are quite weak, Raman spectroscopy has great advantage for detection or discrimination of a trace amount of analytes in aqueous environments. However, Raman scattering cross-sections are inherently small and it generally requires high power excitation and long acquisition times to obtain high-quality Raman spectra. These conditions create disadvantages for the analyses for living cells and real-time monitoring for environmental analyses. Here, I describe a new Raman enhancement technique, namely "electron enhanced Raman scattering (EERS)", where artificially generated electrons additionally affect the polarizability of target molecular systems and enhance their inherent Raman cross-section. Principles of the EERS and its applications to aqueous solutions are presented.
\end{abstract}

(Received February 28, 2007; Accepted March 15, 2007; Published July 10, 2007)

\begin{tabular}{llll}
\hline 1 Introduction & 769 & 5 Summary & 773 \\
2 Brief History of the EERS & 770 & 6 Acknowledgements & 773 \\
3 Principles and Experiments of the EERS & 770 & 7 References & 773 \\
4 Applications & 771 & & \\
\hline
\end{tabular}

\section{Introduction}

Recently, needs for the detection of trace amounts of compounds in complex aqueous media rapidly increase for biomedical and environmental analyses. For these analyses, spectroscopic tools that can distinguish molecules with great similarity are crucial. For such purposes, vibrational spectroscopic techniques based on infrared (IR) absorption or Raman scattering are useful. ${ }^{1}$ They provide information not only on molecular structures but also on local environments of analyte molecules at their functional groups' level. These characteristics allow highlyselective identification and kinetic studies of analytes.

However, detection and identification of trace amounts of molecules in aqueous media by IR absorption have some difficulties. This is because water strongly absorbs IR light. Thus, the weak signal from a trace amount of analyte is often hindered by the strong and broad signals from bulk water. To distinguish signals from objective analyte, we have to remove the contribution from the surrounding enormous amount of bulk water. Raman spectroscopy provides useful tools to detect and identify such minute substances in aqueous media because Raman scattering from water molecules is inherently small. However, cross-sections of Raman processes are generally very small, about $10^{-30} \mathrm{~cm}^{2}$ for a single molecule as compared to that of infrared absorption $\left(10^{-20} \mathrm{~cm}^{2}\right) .^{2} \quad$ To obtain high quality Raman spectra, excitation by high power lasers and long acquisition time are usually required. However, in biomedical analyses, such high power irradiation of laser light to living cells often leads to damage of the cells and analytes. In addition, long acquisition time makes it more difficult to monitor the kinetics and reactions of the analytes in living cells or in the environment in situ.

Because of this situation, enhancement techniques of Raman scattering, especially for the detections in aqueous environments, gain much more importance. This review describes new enhancement techniques for Raman scattering, namely electron enhanced Raman scattering (EERS), a recent approach towards the enhancement of Raman scattering in bulk liquid phase. The EERS effect was found in 1999 when laser-induced plasma was generated in liquid water for elemental analyses of a trace amount of impurities (generally, solid nanoparticles) in highly pure water. In the EERS, transiently generated electrons play a crucial role for the Raman signal enhancement. It should be noted that the EERS requires only electrons and does not require any metal surfaces such as the well-known surface enhanced Raman scattering (SERS). ${ }^{3}$ Thus, the EERS can be applied to various Raman scattering measurements in liquid media. 


\section{Brief History of the EERS}

We found unique Raman enhancement phenomena in laserinduced breakdown (LIB) experiments for analyses of a trace contamination in pure liquid water. ${ }^{4}$ LIB refers to plasma generation by focusing an intense pulsed laser beam in substances. Light emission and supersonic generation following plasma formation are well utilized in spectroscopic analyses and detection. We utilized LIB phenomena for detecting and elemental analyses of trace amounts of small solid nanoparticles in ultrapure water. Threshold energies for LIB are different for solid, liquid, and gas phases. In general, solid materials require lower energy for LIB compared to that of liquid ones. Thus, we can create laser-induced plasma only when solid materials cross the focal point of the laser pulse by controlling the power density of the laser beam. Utilizing the LIB phenomenon, we counted and analyzed small particles in liquid water selectively by supersonic generation and light emission from the plasma of the small particles. Under these experiments, we found that stimulated Raman scattering (SRS) from water molecules was anomalously enhanced only when laser-induced plasma was generated due to the LIB of small solid particles in water. ${ }^{4}$ Interestingly, such enhancement of Raman signals occurred only when laser-induced plasma was generated.

Figure 1 shows a schematic illustration of the laser-induced plasma generation in liquid water and the observation of the SRS in both forward and backward directions. Since laser-

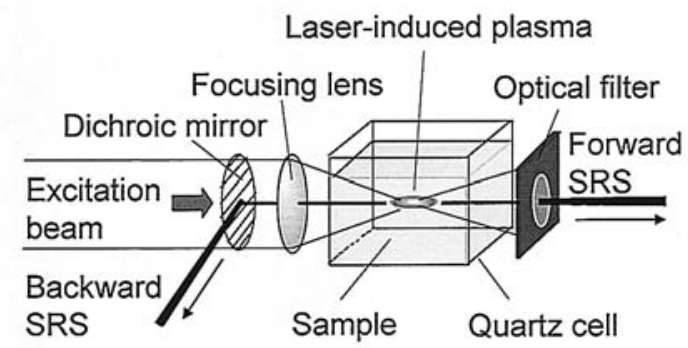

Fig. 1 Schematic illustration of the experimental setup for the SRS measurements from both forward and backward directions with respect to the excitation beam axis. Both forward and backward spectra were measured simultaneously at the same excitation event with a streak camera. induced plasma generation by pulsed laser beam was a single event, we measured the SRS by a streak camera (Hamamatsu Photonics C4334) without accumulation. At the early stage of our LIB experiments, we used a nanosecond pulsed beam of the second harmonics produced from Nd-YAG laser for the plasma generation. ${ }^{4}$ Figure 2 shows the picture of the irradiation of the pulsed beam into a sample cell and the following laser-induced plasma generation in liquid water. When laser-induced plasma was generated in liquid water due to the LIB of solid particles in the focal volume, the bright red spot of the SRS from water molecules was observed in the both forward and backward directions on the optical axis. The peak frequency of the SRS was about $3400 \mathrm{~cm}^{-1}$ and it was attributed to the $\mathrm{OH}$ stretching vibrations of water molecules in liquid phase. However, it seemed to be somewhat curious because the SRS was observed only when the laser-induced plasma was generated, although the power density of the excitation beam was the same at every excitation event. In addition, the SRS peak indicated that the SRS was derived from water molecules in condensed phase, not from gas (plasma) phase, even though the SRS was observed only when laser-induced plasma (gas phase) was generated.

We measured the SRS and plasma continuum emission at the same LIB event and found that the SRS emitted before the onset of plasma continuum emission (Fig. 3). In addition, the duration of the SRS was only several tens of picoseconds for nanosecond excitation (Fig. 4). These results indicated that the conditions for the SRS emission were transiently achieved at the very early stage of the plasma generation and lasted only several tens of picoseconds. The transient conditions in the plasma generation plays a crucial role for the SRS emission, but the water molecules that contribute to the SRS emission remain in the liquid phase.

\section{Principles and Experiments of the EERS}

At the very early stage of the laser-induced plasma generation, electrons are generated by a multi-photon process and the number of the electrons drastically increases by the following avalanche ionization process within a few picoseconds. ${ }^{5}$ Transiently generated electrons should interact with the surrounding water molecules and affect their Raman crosssections through the changes of polarizability. Since the drastically increased electrons strongly scatter the following excitation beam and prevent the transmission of the excitation

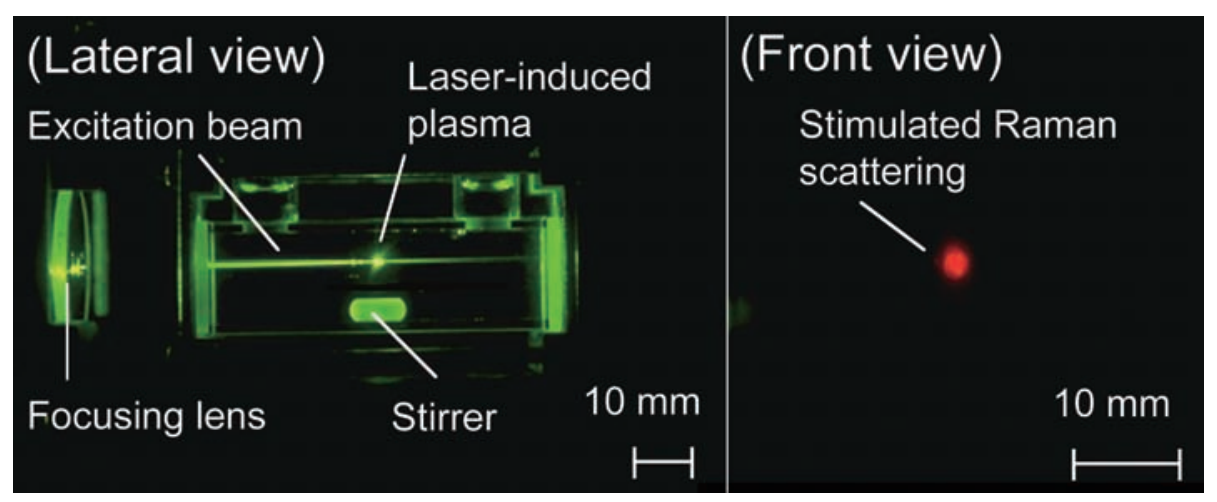

Fig. 2 Pictures of the laser-induced plasma generation in water. Left panel: we can observe a bright spot in the center of the sample cell. The bright spot is white continuum emission from the laserinduced plasma and Rayleigh scattering of the excitation beam. Right panel: we can observe a bright red spot of the SRS both in forward and backward with an optical filter cutting the excitation beam. 


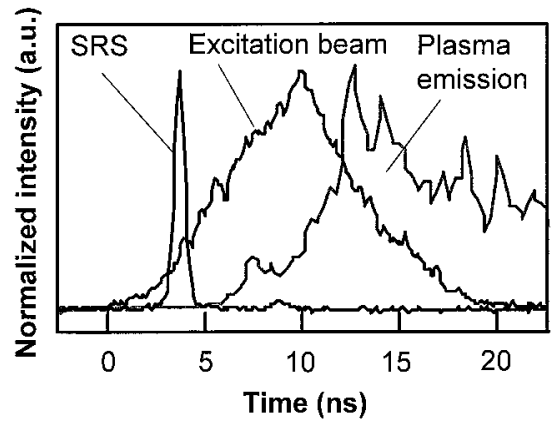

Fig. 3 Temporal profiles of the excitation beam $(532 \mathrm{~nm})$, SRS in the forward direction (wavelength $645-650 \mathrm{~nm}$, Raman shift $\sim 3400$ $\mathrm{cm}^{-1}$ ), and plasma continuum emission (broad band in the visible region; the time profile corresponds to the wavelength $550-600 \mathrm{~nm}$ ) observed at the same excitation event. Plasma continuum emission was observed from the lateral side of the cell. Intensity of each signal was normalized at its maximum intensity.

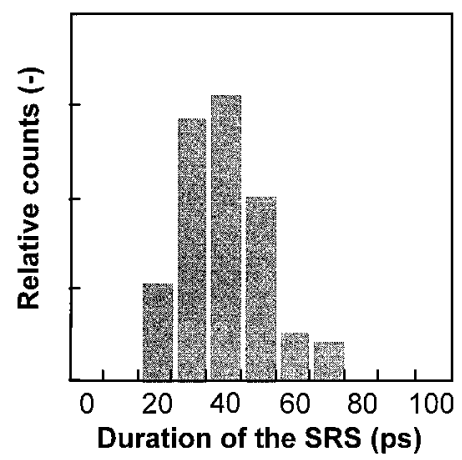

Fig. 4 Histogram of the SRS pulse duration for 12 ns excitation.

beam towards the forward region, such generation and drastic increase of electrons effectively occur in the backward region of the focal volume. To detect the effect of the transiently generated electrons on the Raman cross-section of the water molecules in the backward region of the focal volume, we measured both forward and backward SRS spectra at the same excitation event. Detailed experimental conditions were reported elsewhere. ${ }^{5}$

Figure 5 shows the result. Two characteristic peaks due to the water-electron interactions, which cannot be observed in the SRS spectrum of normal liquid water, were observed in the backward SRS spectrum at 3380 and $3270 \mathrm{~cm}^{-1}$. Interestingly, the electron density was about $10^{20} \mathrm{~cm}^{-3}$ under the present experimental conditions, ${ }^{5}$ namely the number ratio of the water molecules and electrons was about 100:1. The two peaks dominate the backward SRS spectrum. This result indicates that the electrons strongly affect the charge distribution of the water molecules and enhance the Raman cross-section. An enhancement factor of the water molecules-electron system with Gaussian 03 (DFT, 6-31G ${ }^{++}$, B3LYP) was estimated as $10^{3}-$ $10^{5} .7$ We confirmed the electron enhancement effect from the disappearance of such characteristic signals in the presence of electron scavenger $\left(\mathrm{H}_{2} \mathrm{O}_{2}\right)$ in the system.

Such electron-rich water environment was considered to last for several tens of picoseconds until the liquid water was transferred to the plasma (gas) phase by the heating with the following laser pulse. Generation of the electrons and their interactions to water molecules reduced the threshold for the

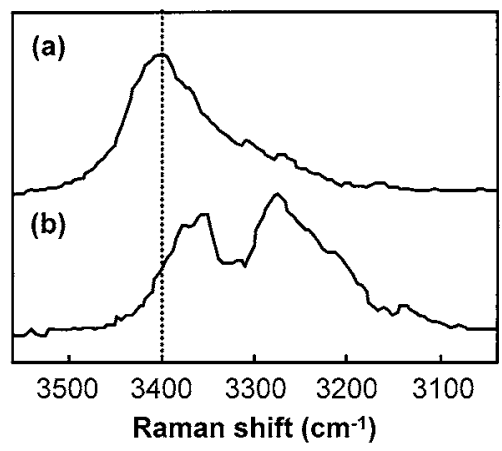

Fig. 5 Comparison of the forward and backward SRS spectra at the same excitation event. The dotted line indicates the normal Raman shift of water in the liquid phase at room temperature.

SRS emission of water molecules by the increase of the Raman cross-section. We denote the Raman enhancement phenomena as electron enhanced Raman scattering (EERS). Effectively generated Raman scattering light stimulates the Raman scattering probability with the existence of the excitation beam, namely a stimulated Raman scattering process is induced. Figure 6 shows the schematic illustration of the time-course of the enhancement mechanism. ${ }^{6}$ It should be noted that observation of the vibrational spectrum of water molecules interacting with electron had been limited to those in gas phase before this observation. The SRS spectrum provides the first vibrational spectrum of the water-electron interactions in condensed phase. The two characteristic peaks should give useful information on the enhancement mechanism and also on the water-electron interactions in liquid phase. This will be discussed in the latter part of the present review.

\section{Applications}

Vibrational spectra in the $\mathrm{OH}$ stretching region (from 3000 to $3800 \mathrm{~cm}^{-1}$ ) sensitively reflect the local hydrogen-bonding network environment. In general, the more the hydrogenbonding network develops, the more the peak frequency of the $\mathrm{OH}$ stretching downshifts. We tested whether the EERS can be applicable to the analyses of local environments in aqueous solutions.

At first, interactions between water molecules and electrons in liquid phase will be discussed. The aqueous or hydrated electron is a matter of great interest in physical chemistry, radiation chemistry, and bio- and environmental chemistry where the electrons in water play an important role for chemical reaction or energy transfer. Since the first spectral identification of the aqueous or hydrated electron in $1962,{ }^{8}$ its physical or chemical properties have been intensively studied from both experimental and theoretical aspects. Electrons in liquid water have been detected by visible absorption spectra, but water molecules interacting with electrons in liquid phase have not been detected in the vibrational spectral region. The EERS spectra in the $\mathrm{OH}$ stretching region are expected to provide detailed information on the water molecules interacting with electrons in the liquid phase. As mentioned, two characteristic peaks due to the interaction between water molecules and electrons were observed in the backward EERS spectrum. The frequency of the two peaks agreed well with that observed in water cluster anions $\left(\mathrm{H}_{2} \mathrm{O}\right)_{\mathrm{n}^{-}}(n=6,7) .^{9}$ In liquid phase, electron is considered to be localized and supported within a 
(a)

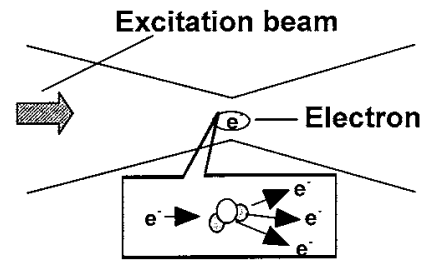

(b)

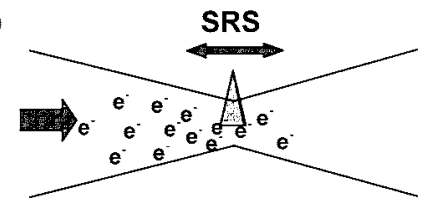

(c)

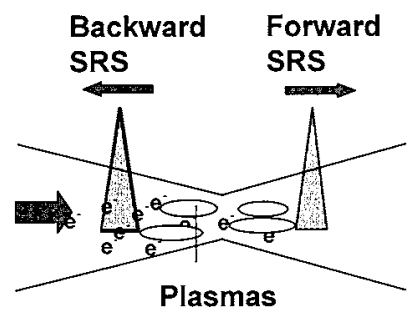

(d)

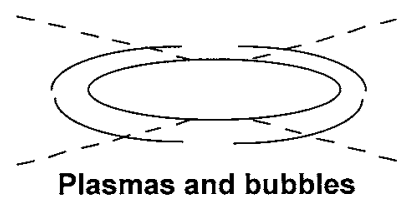

Fig. 6 Schematic illustration of the mechanism for the transient SRS enhancement of the SRS. (a) At the very early stage of the pulsed beam irradiation, small solid impurities (nanoparticles) or water molecules ejected electrons by multi-photon excitation. The number of the electrons was drastically increased in a few picoseconds through the inverse bremsstrahlung by the strong laser field and avalanche ionization due to the collision between accelerated electrons by the strong laser field and molecules. (b) Increase of the electrons in the focal region. Due to the strong scattering of the excitation beam by the electrons at the center of the focal region, more electrons are generated in the backward region than in the forward region. Raman scattering cross section of water molecules are transiently enhanced, resulting in the onset of the SRS (c) The generated SRS growths both in forward and backward direction by the coherent interactions with the strong laser field of the excitation beam. During several tens of picoseconds, water in the focal region transferred to the plasma state. The phase transfer strongly perturbs the coherent growth of the SRS. (d) Plasma recombination and bubble generation.

cavity formed by surrounding water molecules. ${ }^{10,11}$ Corresponding interactions between water molecules and electrons also occur in bulk water systems. The lower frequency peak observed at $3270 \mathrm{~cm}^{-1}$ disappeared at higher temperature region $(>340 \mathrm{~K})$. To the contrary, the higher frequency peak observed at $3380 \mathrm{~cm}^{-1}$ remained in the higher temperature. Although detailed structures of trapping sites for electrons have not been elucidated in the condensed phase, the different temperature dependence of the two peaks indicates that at least two distinctive interactions exist between the water molecules and electrons in the liquid phase.

Next, we applied the EERS to water/ethanol binary solutions. In dilute ethanol aqueous solution, the partial molar volume of water is known to decrease. However, it has been difficult to detect sensitively the slight changes of the hydrogen-bonding network environment induced by a small amount of ethanol

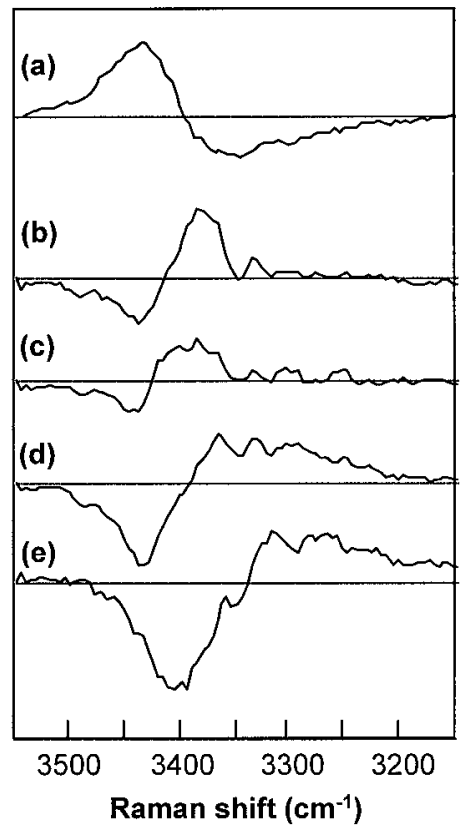

Fig. 7 (a) Difference EERS spectrum between $1 \mathrm{M} \mathrm{KCl}$ aqueous solution and bulk water. (b) - (e) Difference EERS spectra between ethanol aqueous solution and bulk water. Ethanol concentration: (b) 1, (c) 2, (d) 3, (e) 4 mol\%.

molecules embedded in a hydrogen-bonding network of bulk water. Thus, the local hydrogen-bonding network environment of the hydration structure in dilute ethanol aqueous solution has remained as an intriguing topic. Since EERS gives enhanced Raman signals in liquid phase, we expected that the EERS would provide further information on the slight change of local hydrogen-bonding network environment in hydration area. To test whether the EERS can detect the changes in hydrogenbonding network environment, $\mathrm{KCl}$ aqueous solution was measured first. $\mathrm{KCl}$ is well-known as a structure-breaking solute. We can expect that $\mathrm{KCl}$ breaks or weakens the hydrogen-bonding network of water, leading to the peak frequency shift towards the higher side compared to that in pure water, as is observed by normal Raman spectrum measurements. Figure 7(a) shows the difference EERS spectrum between $\mathrm{KCl}(1 \mathrm{M})$ aqueous solution and bulk water. As expected, the peak shift was observed due to the change in local hydrogen-bonding network environment. Then we applied EERS to analyze dilute ethanol aqueous solution. ${ }^{12}$ Figures 7(b) - (e) show the difference EERS spectra between ethanol aqueous solutions and bulk water. In general, hydrophobic hydration is achieved by development of an ice-like hydrogenbonding network around hydrophobic groups (here, alkyl chains of ethanol). If such an ice-like hydrogen-bonding network is developed, an intensity increase in the lower frequency region is expected. However, such a feature indicating the development of an ice-like structure was not observed. In contrast, we can observe a sharp decrease in the higher frequency region. These changes in the EERS spectra did not give any evidences for the development of an ice-like structure, but showed that some reinforcement on the hydrogen-bonding network occurred due to adding a small amount of ethanol molecules. Flexible participation of hydroxyl groups of the ethanol molecules into the original hydrogen-bonding network between water molecules seems to contribute to reduce defects or strongly distorted and weak hydrogen-bonds in liquid water, resulting in 

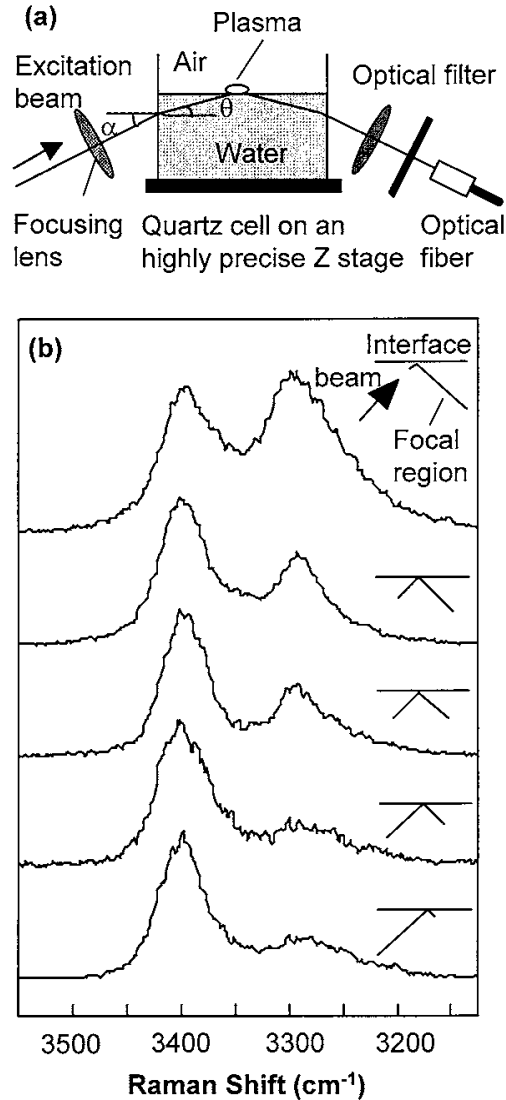

Fig. 8 (a) Schematic illustration of the ATR-EERS experiment The incident angle of the excitation beam to the air/water interface $(\theta)$ is $8^{\circ}\left(\alpha: 10.5^{\circ}\right)$. (b) The ATR-EERS spectra measured at different positions of the focal region stretched along the beam axis across the air/water interface.

the reinforcement of the hydrogen-bonding network of liquid water.

Then we applied the EERS to interfacial water. ${ }^{13}$ Interfacial water plays an important role in molecular transfer that occurs at air/water interfaces in the environment, in electrochemical reactions, and elsewhere. To monitor dynamic chemical processes that occur at interfacial regions, one requires highly sensitive spectroscopic tools because the total amount of analyte in the interfacial region is quite small due to the space restriction. In addition, to probe chemical reactions, vibrational spectra are highly useful because they provide detailed information on the changes of functional groups of analytes. In general, to obtain vibrational spectra of the interfacial area, the sum frequency generation (SFG) technique, one of the nonlinear spectroscopy, is suitable. However, to obtain SFG spectrum, one needs a tunable IR laser system and a long acquisition time.

Laser-induced plasma was generated at an air/water interface by focusing an excitation beam onto the interface under the attenuated total reflection (ATR) condition (ATR-EERS, Fig. 8(a)). Figure 8(b) shows the ATR-EERS spectra of the air/water interface. We can observe a characteristic peak at around $3200 \mathrm{~cm}^{-1}$, which can not be observed in normal Raman spectrum from bulk water. From the comparison with the sum frequency generation (SFG) spectra of air/water interfaces, the characteristic peak was assigned to a quasi ice-like hydrogenbonding network environment, which is characteristic at the air/water interface. It is worth noting that the spectrum was obtained by a single excitation event using the ATR-EERS technique. However, another characteristic peak at $3700 \mathrm{~cm}^{-1}$, representing dangling $\mathrm{OH}$ groups at the interface, was not observed in the ATR-EERS spectrum. In the ATR-EERS experiment, many electrons are generated at the air/water interface. Such electrons should strongly interact with positively polarized hydrogen atoms of the dangling $\mathrm{OH}$ groups at the interface. These interactions may result in termination of the dangling $\mathrm{OH}$ groups. This result may include interesting information from the viewpoint of investigation on plasma/water interfaces, which have not been clarified yet. Although further investigation should be required to develop the ATR-EERS to a conventional analytical tool for interfacial water, the ATR-EERS has a potential to be a novel spectroscopic tool for obtaining information on interfacial water environments with short accumulation time and simple optical configuration.

In the present review, the applications of the EERS for aqueous solutions were described. The EERS can be also applied in organic systems and enhancement of $\mathrm{CH}$ stretching mode was observed in benzene/toluene binary system. ${ }^{14}$ Recently, we applied the EERS technique to analyze local environment of water molecules in supercritical conditions; details will be reported elsewhere. ${ }^{15}$

\section{Summary}

New enhancement technique of Raman scattering in bulk liquid phase was reviewed. Transiently generated electrons and their interactions to analytes are responsible for the enhancement of the Raman signals. The enhancement effect was denoted as electron enhanced Raman scattering (EERS). The EERS was applied to study water-electron interactions in the liquid phase, and hydrogen-bonding network structures of dilute ethanol solutions. The ATR-EERS was also developed and was tested to obtain information on interfacial water. In the previous EERS, electrons are generated by laser-induced plasma generation. Although the EERS signals came from the liquid phase, namely before the phase transition from liquid states to plasma phase, plasma generation gives damages to the sample and inhibits the signal accumulation. Thus, combinations of the EERS with simple and milder electron generation techniques are expected to provide generalized Raman enhancement tools for the analyses of solutions in various, complicated liquid environments such as in living cells. Further instrumental development of the EERS and its applications are now underway.

\section{Acknowledgements}

Prof. Tsuguo Sawada and Prof. Takahiko Kitamori (The University of Tokyo) were very helpful as supervisors of the present research. I thank Prof. Kimihiko Hirao and Dr. Takahito Nakajima (The University of Tokyo) for their cooperation with the theoretical calculation of the enhancement factor. Dr. Kazuo Terashima and Mr. Takaaki Tomai (The University of Tokyo) showed great cooperation during the analysis of the water-electron interactions in supercritical water.

\section{References}

1. J. M. Chalmers and P. R. Griffith, "Handbook of Vibrational Spectroscopy", 2001, John Wiley \& Sons, 
Chichester, UK.

2. N. Hayazawa, Y. Inouye, Z. Sekkat, and S. Kawata, J. Phys. Chem., 2002, 117, 1296.

3. G. A. Baker and D. S. Moore, Anal. Bioanal. Chem., 2005, 382, 1751.

4. H. Yui, Y. Yoneda, T. Kitamori, and T. Sawada, Phys. Rev. Lett., 1999, 82, 4110.

5. H. Yui and T. Sawada, Phys. Rev. Lett., 2000, 85, 3512.

6. H. Yui and T. Sawada, Rev. Sci. Instrum., 2003, 74, 456.

7. H. Yui, T. Nakajima, K. Hirao, and T. Sawada, in preparation.

8. E. J. Hart and J. W. Boag, J. Am. Chem. Soc., 1962, 84, 4090.

9. C. G. Bailey, J. Kim, and M. A. Johnson, J. Phys. Chem.,
1996, 100, 16782.

10. D. H. Paik, I.-R. Lee, D.-S. Yang, J. S. Baskin, and A. H. Zewail, Science, 2004, 306, 672.

11. M. J. Tauber and R. A. Mathies, J. Am. Chem. Soc., 2003, 125, 1394.

12. H. Yui, H. Kanoh, H. Fujiwara, and T. Sawada, J. Phys. Chem. A, 2002, 106, 12041.

13. H. Yui, H. Fujiwara, and T. Sawada, Chem. Phys. Lett., 2002, 360, 53.

14. H. Yui, T. Nakajima, K. Hirao, and T. Sawada, J. Phys. Chem. A, 2003, 107, 968.

15. H. Yui, T. Tomai, M. Sawada, and K. Terashima, in preparation. 